\title{
The ribosome returned
}

\section{Peter B Moore}

Address: Department of Chemistry, Yale University, New Haven, CT 06520-8107, USA. Email: peter.moore@yale.edu

Published: 26 January 2009

Journal of Biology 2009, 8:8 (doi: I0.1 I86/jbiol I03)

The electronic version of this article is the complete one and can be found online at http://jbiol.com/content/8/I/8

(c) 2009 BioMed Central Ltd

\begin{abstract}
Since the mid-1990s, insights obtained from electron microscopy and X-ray crystallography have transformed our understanding of how the most important ribozyme in the cell, the ribosome, catalyzes protein synthesis. This review provides a brief account of how this structural revolution came to pass, and the impact it has had on our understanding of how the ribosome decodes messenger RNAs.
\end{abstract}

About 20 years ago, for reasons now lost in the mists of the 20th century, I wrote a review about the ribosome for Nature [1]. Ribosomes had been discovered in the mid1950 s and, until the late 1960s, ribosome research was a major part of molecular biology. By the late 1960s it had emerged that ribosomes are the polymerases that catalyze protein synthesis under mRNA control. Satisfied with that level of understanding, most who had worked on protein synthesis during the 'golden age' of molecular biology sought greener pastures in the years thereafter, and interest in the ribosome waned. The thesis of my review, which was entitled 'The ribosome returns', was that the ribosome field was poised for advances so dramatic that it would regain the prominence it had last enjoyed in the mid-1960s.

In 1988 there were two reasons for optimism. First, the discovery of ribozymes in the late 1970s had stimulated the interest of biochemists and molecular biologists in RNAcontaining objects generally, and the ribosome is the most important RNA-containing object of them all. Second, the shortage of structural information that had for so long plagued the ribosome field seemed ready to end.

A month or so ago, I agreed to write a successor to "The ribosome returns' for Journal of Biology, but shortly thereafter I started having second thoughts. As Yogi Berra is alleged to have said, "It is hard to make predictions, especially about the future". By writing a successor to "The ribosome returns' I would be in the embarrassing position of calling attention to an ancient review, the very title of which was a prediction. Below I provide a personal account of what happened in the ribosome field between 1988 and 2000 and my assessment of where the field stands today. As it happens, the ribosome did return, but it took a while.

\section{How the structural drought ended}

By 1988, a lot had been learned about the three-dimensional organization of the ribosome. The shapes of the two ribosomal subunits, and of the complex they form during protein synthesis, were known at low resolution (Figure 1), and it was understood that protein synthesis occurs in the gap between the two subunits. Much had been learned about the placement of ribosomal proteins within those shapes. The secondary structures of the ribosomal RNAs (rRNAs) had been worked out, and sites on rRNAs where ribosomal proteins bind had been identified. In addition, the structures of several ribosomal proteins and a few rRNA fragments were known at atomic resolution in isolation. However, no one was so deluded as to imagine that 


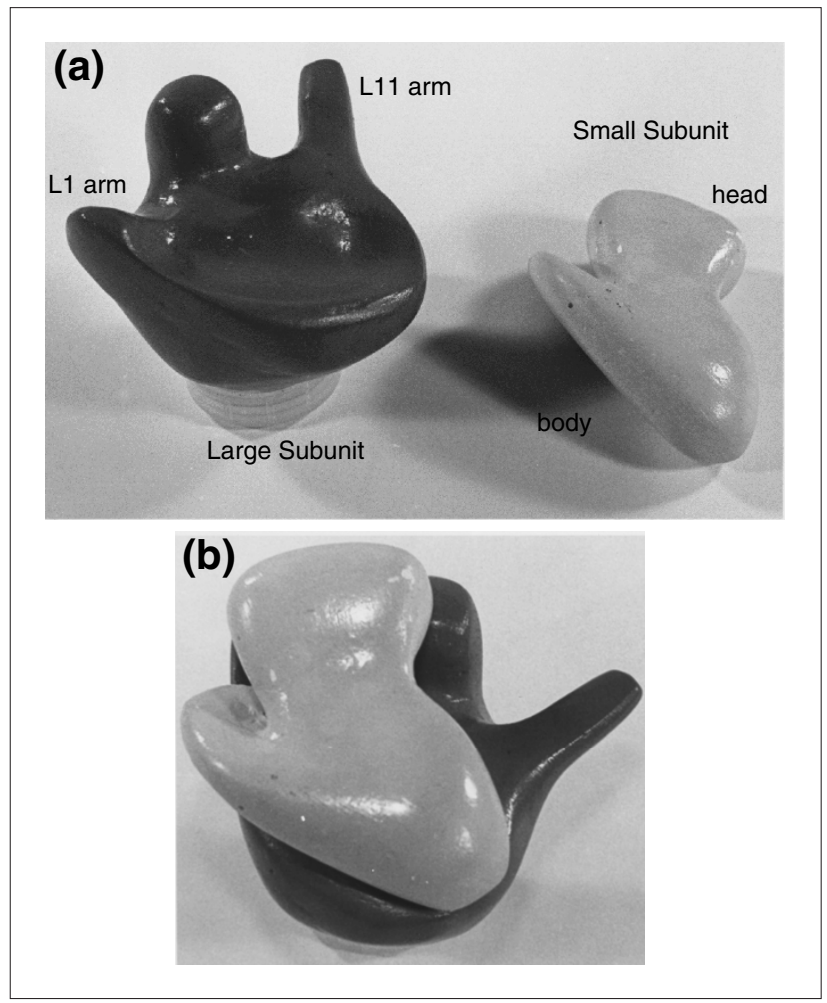

Figure I

The ribosome at low resolution. The images shown here are photographs of plaster models of the two ribosomal subunits made by James Lake. They were derived from his EM images of the two ribosomal subunits from $E$. coli [45]. The resolution is about $40 \AA$. (a) The large subunit (left) and the small subunit (right) with some of their landmarks indicated. (b) The arrangement of the two subunits in the complete ribosome.

structural information of this sort would ever explain ribosome function.

The only two approaches for addressing the need for structural information that seemed promising in the $1980 \mathrm{~s}$ were X-ray crystallography and electron microscopy. The first ribosome crystals, reported by Yonath, Wittmann and colleagues in 1980 [2], diffracted poorly; but, as the years went by, crystals were obtained of ribosomes and ribosomal subunits from many prokaryotic species (for example [3]), and the resolutions of the diffraction patterns of the best of them improved.

The unit cells of ribosome crystals are very large and, consequently, ribosome crystals diffract X-rays so weakly that useful data can be collected from them only at synchrotron light sources. In 1980 the technology for doing macromolecular crystallography at synchrotrons was primitive, but major advances were made in the 1980s and thereafter (for example [4,5]), and by 1988 the technology needed for ribosome crystallography was falling into place.

Electron microscopy seemed promising because methods were being developed for obtaining three-dimensional electron density maps of biological objects from their twodimensional electron microscopic (EM) images [6]. Although the theory of image reconstruction is simple, its application to objects like the ribosome, for which the images to be reconstructed are those of isolated, randomly oriented particles, was fraught with difficulties. Nevertheless, by 1988 it seemed likely that ribosome reconstructions would eventually emerge with resolutions high enough to allow tRNAs to be visualized bound to the ribosome. Once that threshold was crossed, it seemed to me that EM would start contributing to our understanding of protein synthesis.

My optimism notwithstanding, nothing published between 1988 and 1995 would have led the unbiased observer to conclude that the ribosome was likely to return any time soon. The advances made in EM reconstructions did not seem dramatic, and the papers published on ribosome crystallography were records of frustration. Ribosome crystallography had run aground on the shoals of the classic problem in macromolecular crystallography, the so-called phase problem, and it was unclear if it would ever get unstuck.

Crystal structures are three-dimensional models of molecules that are generated by fitting chemical structure into experimentally determined, three-dimensional maps that display the distributions of electrons in those molecules. Electron density maps can be computed from crystal diffraction data only if the phases associated with each of the tens of thousands of reflections in such datasets are known. If there is no prior knowledge about the threedimensional structure of a macromolecule, phases must be measured experimentally. In the end, the experimental technique that contributed the most to solving ribosome crystal structures was the heavy atom multiple isomorphous replacement (MIR) method that Perutz devised in the 1950s to solve the structure of hemoglobin. However, in 1988, it was unclear that MIR, or any other approach to phasing, such as anomalous scattering (AS), would ever work for the ribosome. Everything else being equal, the larger a macromolecule, the harder it is to phase its diffraction pattern experimentally; and by crystallographic standards, ribosomes were and are huge.

Ad hoc ribosome meetings have been held at different venues around the world for decades. In my estimation, none of them was more important than the ribosome conference that took place in Victoria, BC, Canada, in the summer of 1995. There, Frank and his colleagues presented 
the reconstructions they had just obtained from EM images of ribosomes embedded in vitreous ice [7]. Even though the resolutions of these reconstructions were modest by today's standards, about $25 \AA$, their superiority over their predecessors, which had been derived from images of negatively stained particles, was striking. The electron microscopy of the ribosome had just taken a great leap forward.

No significant progress was reported on the crystallographic front at Victoria [8], but it was clear that this area too was heating up. A group at Yale, of which I was a member, had just begun working with ribosome crystals, and gossip at the meeting revealed that we were not alone. Most notably, by the time I left Victoria, I was convinced that the ribosome phase problem was soluble. Cryo-EM structures might do the job. Molecular replacement is a computational method for using the structure of one macromolecule to estimate the phases of the reflections in the diffraction pattern produced by a crystal of another macromolecule of related structure. Why not use a cryo-EM reconstruction of the ribosome to phase ribosome diffraction patterns by molecular replacement? Although this approach would yield phases only up to the resolutions of the EM reconstruction used, which was likely to be low by crystallographic standards, once the proverbial foot was in the proverbial door that far, higher resolution electron density maps would surely follow.

It still took a while for ribosome crystallographers to obtain the phases they needed. The first successful phasing of a ribosomal diffraction pattern was reported in 1998 [9]. That paper presents a $9 \AA$ resolution electron density map of the large ribosomal subunit from Haloarcula marismortui, which was obtained using crystals that had been first described 13 years earlier [10]. The phases used to compute that map were measured by MIRAS methods using crystals into which heavy metal cluster compounds had been soaked. The crucial step in all phasing experiments is determination of the locations in the unit cell where heavy metals and/or anomalous scatters reside. This is normally done using Patterson methods, but in this instance, in order to prove that these sites had been correctly located, a second, independent approach was used. Phases were obtained by molecular replacement using an EM electron density map of the $H$. marismortui large ribosomal subunit. Using those phases, a difference electron density map was computed that displayed only the electrons belonging to the heavy atoms in the unit cell. The Patterson-derived positions for these heavy atoms corresponded exactly to the positions found by molecular replacement. Even though little molecular detail can be made out in any $9 \AA$ resolution electron density map, the fundamental accuracy of this $9 \AA$ resolution electron density map was beyond question.
Because nothing motivates scientists more powerfully than the knowledge that their problem can be solved, ribosome crystallography advanced rapidly thereafter. By the summer of 1999, the resolution of the electron density maps available for the large ribosomal subunit from $H$. marismortui had improved to $5.0 \AA$ [11], and using heavy atom isomorphous replacement methods, Ramakrishnan and colleagues had obtained a $5.5 \AA$ resolution electron density map for the small ribosomal subunit from Thermus thermophilus [12]. A $7.5 \AA$ resolution electron density map of the 70 S ribosome from $T$. thermophilus appeared that same year [13].

The crystallographic drama came to a climax in 2000. In August, a $2.4 \AA$ resolution structure was published of the large ribosomal subunit from $H$. marismortui [14]. (Figure 2 shows what the improvements in the resolutions of the electron density maps for this object between 1998 and 2000 meant in terms of their interpretability.) At the beginning of September, an imperfect, $3.3 \AA$ resolution structure of the small ribosomal subunit from $T$. thermophilus was presented [15], and 3 weeks thereafter a significantly more accurate, $3.1 \AA$ resolution version of that same subunit appeared that had been independently determined [16].

\section{What is known about ribosome structure today?}

Since 2000, many ribosome crystal structures have been deposited in the Protein Data Bank (PDB). I think of seven of them as founder structures: that is, the first atomic resolution structure obtained from a particular ribosome crystal by a particular laboratory. The three structures that appeared in 2000 are founder structures as are, first, a structure of the large ribosomal subunit from Deinococcus radiodurans [17], second, two independently determined structures for the 70S ribosome from T. thermophilus [1820], and third, a structure of the $70 \mathrm{~S}$ ribosome from Escherichia coli [21]. The rest of the ribosome structures in the PDB are those of founder particles with substrates, substrate analogs, protein factors and inhibitors bound, and all of them were generated using effectively the same crystals that produced the corresponding founder structures. (Once the structure of some crystal has been solved, experimental phase determination is not required to solve the structures of derivatives of that crystal.)

Since 2000, the goal of ribosome crystallographers has been the construction of a movie of protein synthesis, the individual frames of which are atomic resolution structures of the ribosome in every state it visits as protein synthesis progresses. This movie is far from complete, mostly because the crystals needed to determine most of its frames are still not available; but it is not for lack of trying. 


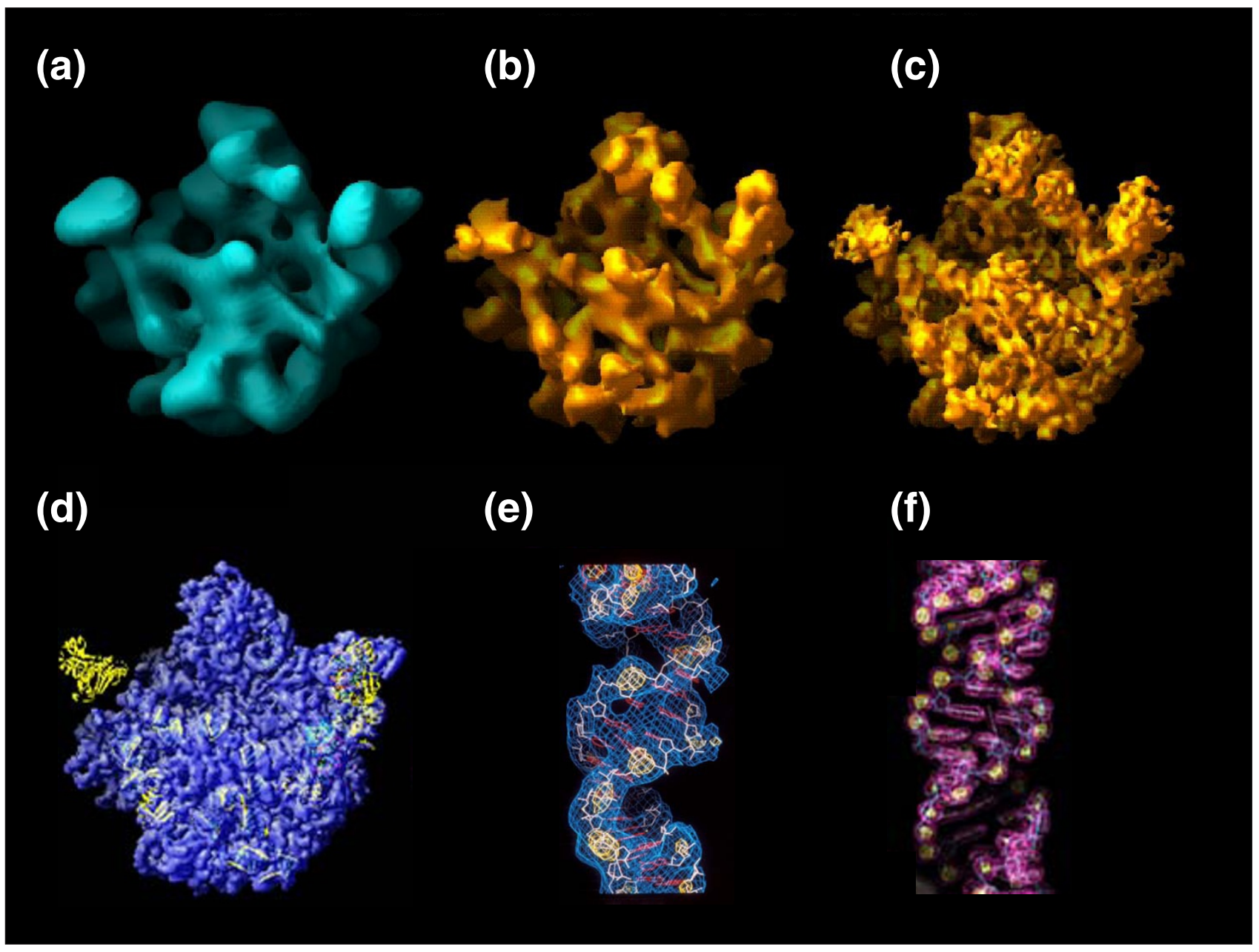

Figure 2

The effect of improvements in resolution on ribosome electron density maps. (a-c) Views of the entire surface of the large ribosomal subunit of H. marismortui that interacts with the small ribosomal subunit. (a) An EM reconstruction of that subunit that has a resolution of $20 \AA$. (b) An X-ray crystallographic image of the same particle, also at a resolution of $20 \AA$. (c) The same as (b) except for the resolution, which is $9 \AA$. All three images in (a-c) are from [9]. (d) A view of the face of the large subunit at a resolution of $5 \AA$, with the positions of LI and LIO indicated in yellow [II]. (e) Electron density corresponding to a helix of $23 \mathrm{~S}$ rRNA at a resolution of $5 \AA$ [ I I ]. (f) The electron density for a helix at a resolution of $2.4 \AA$ [14].

Besides not having all the frames needed to make the complete movie of protein synthesis, what else do we not know about ribosomes crystallographically? In the first place, there are no crystal structures for eukaryotic ribosomes, and in a world controlled by sponsors fixated on Homo sapiens this is not a good thing. In addition, surprisingly, there is still no complete crystal structure for a large ribosomal subunit. Neither of the two lateral arms of the large ribosomal subunit (Figure 1) can be visualized at high resolution in crystal structures of isolated large subunits; they wiggle too much. The conformation of the L1 arm is stabilized when the small subunit binds to the large and, for that reason, its structure is known in at least some of the conformations it adopts during protein synthesis. Much less is known about the structure of the L11 arm. The L11 protuberance includes the complex that ribosomal protein L10 forms with four to six copies of ribosomal protein L7/L12 (the number depends on species [22]). The L10 complex is very important functionally, but only its L10 portion has ever been visualized crystallographically, even partially [23], and its distal components are so flexible that they may never be visualized, either crystallographically or by EM. However, structures are available for much of the L10 complex in isolation, and when those structures are added to the structure of the rest of the large subunit structure in silico, the effect is startling, to put it mildly [24] (Figure 3). We have no idea what this part of the ribosome does to promote protein synthesis. 


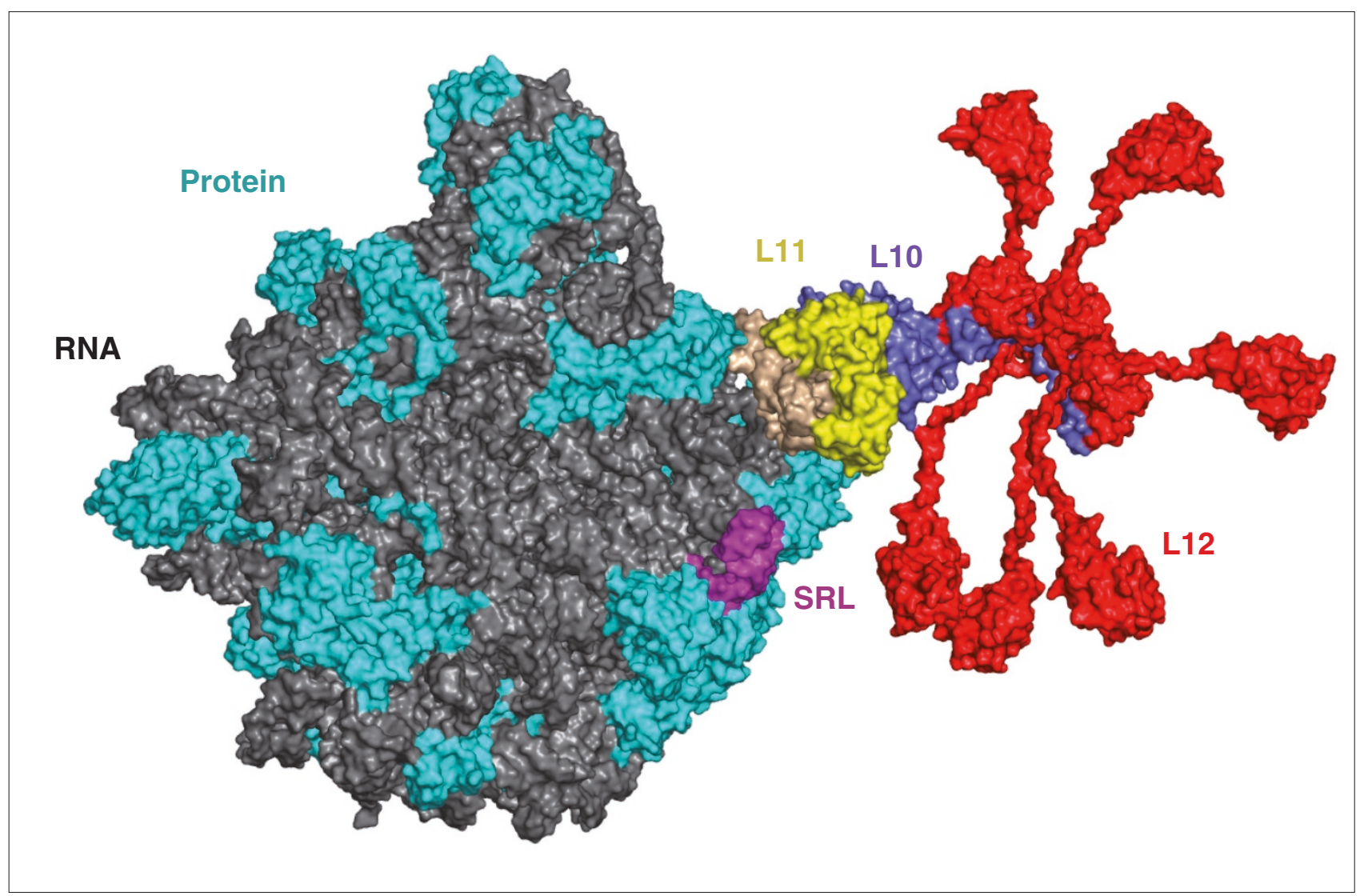

\section{Figure 3}

The LI I arm of the large ribosomal subunit. There are structures available for most of the complex that LI0 forms with the several copies of L7/LI2 that interact with it in the ribosome as isolated proteins. These structures have been added to the structure of the large ribosomal subunit from H. marismortui in silico [24]. Modified from [24].

The $30 \mathrm{~S}$ subunit is less problematic structurally. The small ribosomal subunit is much more dynamic conformationally than the large ribosomal subunit, and its flexibility is vital for its function (see below), but we have crystal structures for the entire object in several of its states.

It is much easier to study the functional complexes of the ribosome by electron microscopy than it is to investigate them crystallographically. Electron microscopists do not need crystals, and the amounts of material they consume are orders of magnitude less than crystallographers require. Finally, image sorting techniques now exist that make it possible to obtain reconstructions of ribosomes in specific functional states, starting with images of samples in which only a fraction of the particles present are in that state; pure samples are no longer required. Consequently, as far as the making of the movie of protein synthesis is concerned, the electron microscopists are well ahead of the crystallographers and, in addition, EM images of eukaryotic ribosomes exist (for example [25]).
Three-dimensional electron density maps obtained by EM are qualitatively similar to crystallographic electron density maps, and they are commonly used to generate (quasi) atomic resolution models of ribosomes, even though none of them has a resolution high enough to allow an atomic resolution model of the ribosome to be constructed from it de novo. EM-derived models of the ribosome are generated by fitting crystal structures into EM electron density. This procedure leads to difficulties only in those parts of a structure where you are most in need of information, namely where the structure of the EM object diverges from the crystal structure(s) used to model it.

The other major problem faced by consumers of EM images is variation in resolution, which makes comparison of images difficult. Since 1995, substantial advances have been made in image reconstruction, and newer reconstructions generally have higher resolutions than older reconstructions. One reason is that the labor per image required to generate a reconstruction has fallen, and resolution improves 
as the number of images merged grows. Molecular properties can also limit resolution. The reconstruction process assumes that the images under analysis are different views of the same three-dimensional object. No increase in the number of images processed can overcome resolution limitations caused by particle-to-particle variations in structure, unless the variation involves discrete conformational states, in which case image sorting may save the day.

\section{Structure and function during the elongation phase of protein synthesis}

Like Gaul, protein synthesis divides naturally into three parts: initiation, elongation and termination (Figure 4). During initiation, the two subunits of the ribosome are assembled into a complex that has an aminoacylated initiator tRNA and an mRNA bound, ready to make the first peptide bond of a protein [26,27]. During termination, a completed protein is released from its tRNA, and the ribosome assembly dismantled so that its components can be recycled $[28,29]$. The steps of the elongation phase, which is the part of the process we will discuss here, constitute a cycle that must be repeated for every peptide bond formed. (A movie showing the three phases of protein synthesis has been created by the Ramakrishnan group at the MRC Laboratory of Molecular Biology and can be accessed from their website [30].) The purpose of the references provided here, which are entirely to recent articles and reviews, is to give the reader an entrée into the relevant literature, rather than an outline of the history of the field.

The ribosome catalyzes two chemical reactions: the aminolysis of the ester bonds that link nascent polypeptides to tRNAs during protein synthesis, and the hydrolysis of those same bonds. The amino group used for the aminolysis reaction is the $\alpha$-amino group of an amino acid that is esterlinked through its $\alpha$-carboxyl group to the $3^{\prime} \mathrm{OH}$ of the $3^{\prime}$ terminal nucleotide of a tRNA. The products of this reaction are a peptide that is one residue longer than it was before the reaction, ester-linked to the tRNA that carried the amino acid into the reaction, and a tRNA that has nothing attached to it. Because this reaction transfers a peptide from one tRNA to another, it is referred to as the peptidyl transferase reaction. In the second reaction, which occurs only during the termination phase of protein synthesis, the nucleophile is water, instead of an $\alpha$-amino group, and peptides are transferred to water (that is, the last tRNA is released from the newly synthesized protein).

Both the peptidyl transferase reaction and the hydrolysis reaction occur at the same site on the ribosome, its peptidyl transferase center (PTC), about which we now know quite a lot. Our understanding can be summarized as follows [31].

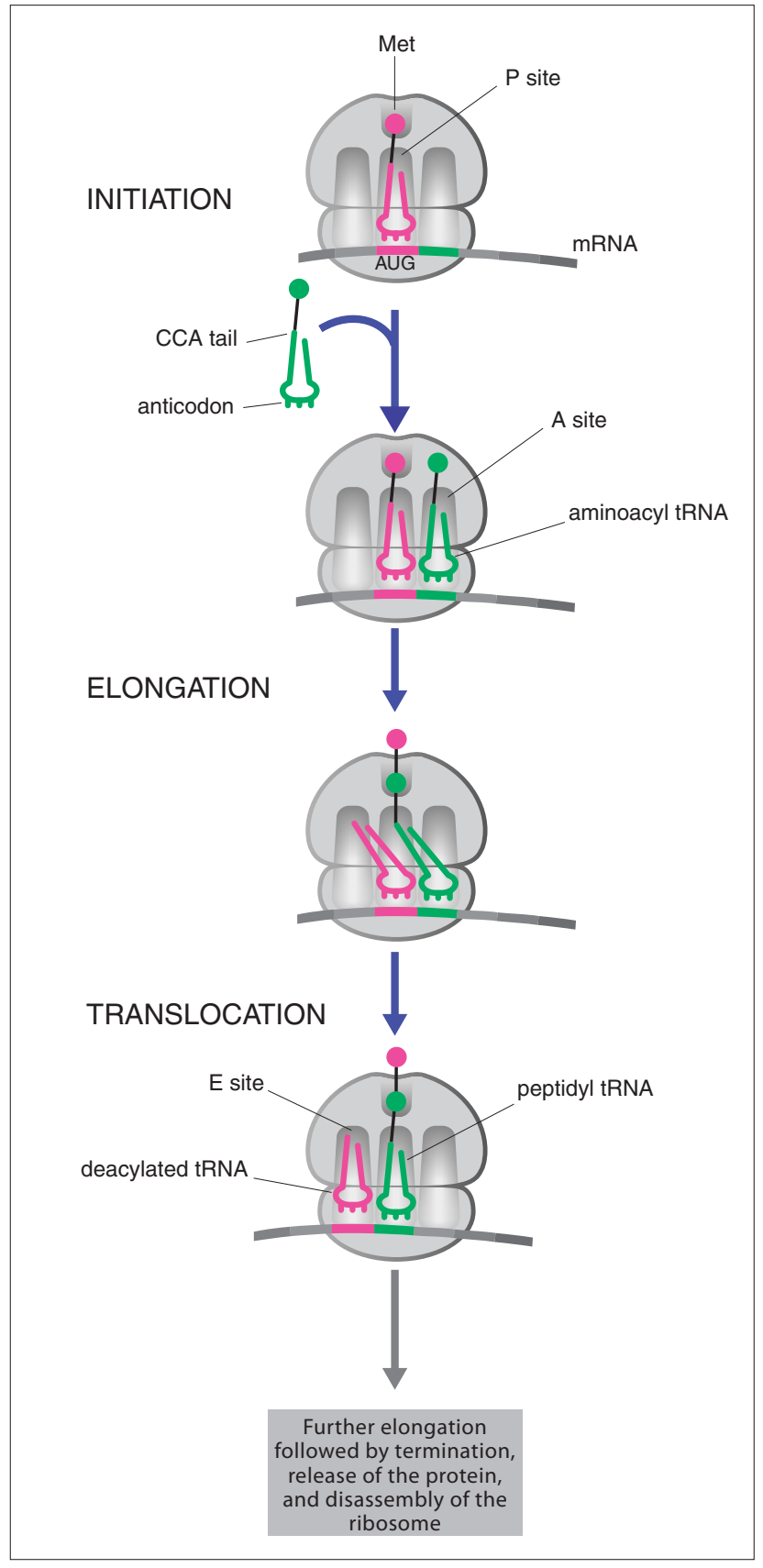

Figure 4

The translation cycle. The intiation of translation is complete once an aminoacyl tRNA charged with formylated methionine has been placed in the $P$ site bound to the initiating AUG codon of an mRNA. Elongation begins when a second aminoacyl tRNA recognizes its cognate codon and binds in the A site. This is followed by transfer of the amino acid from the tRNA in the P site to form a peptide bond with the amino acid attached to the tRNA in the A site, and translocation of the two tRNAs into the $E$ and the $P$ site, respectively. This sequence is iterated until a stop codon is encountered, the completed protein is released, and the ribosome disassembles, at termination of the translation cycle (not shown). 
(1) The PTC is located in the center of the subunit interface surface of the large ribosomal subunit. (2) Although RNAprotein interactions are essential for stabilizing the conformation of the PTC, it is composed entirely of RNA: the ribosome is a ribozyme. (3) The catalytic properties of the PTC are not modulated by interactions between the two subunits. (4) The PTC includes a site that accommodates peptidyl tRNAs, the $\mathrm{P}$ site, and a site to which aminoacyl tRNAs bind, the A site (see Figure 4). (5) Both the A site and the P site of the PTC interact primarily with the 3 ' terminal CCA sequence that is common to all tRNAs. Thus, to first approximation, differences in tRNA sequences make no difference in the PTC. (6) When the A site of the PTC is empty and its P site is occupied, the PTC adopts a conformation that protects the ester bonds of peptidyl tRNAs from nucleophilic attack. A conformational change accompanies the binding of aminoacyl tRNAs to the A site (as well as the binding of release factors to the ribosome) that exposes the ester bond in the P site to nucleophilic attack. (7) Beyond positioning substrates properly, the PTC seems to do little to enhance the rate of peptide bond formation. (8) The group that makes the largest chemical contribution to the rate of the peptidyl transferase reaction is the $2^{\prime} \mathrm{OH}$ of the $3^{\prime}$-terminal A of the tRNA in the P site, which facilitates the removal of a proton from the attacking amino group and the addition of a proton to the leaving group, which is the 3' $\mathrm{OH}$ of the tRNA bound in the $\mathrm{P}$ site. (9) On the ribosome, the peptidyl transferase reaction proceeds at a rate that is about $10^{7}$ times faster than the rate of similar reactions in solution. (10) Once substrates are bound appropriately to the PTC, the peptidyl transferase reaction occurs at a rate that is at least ten times faster than the overall rate of protein synthesis in living cells, which is about $20 \mathrm{~s}^{-1}$. (11) At neutral $\mathrm{pH}$, the ester bond of an aminoacyl tRNA is a high energy bond, but the ester bond in a peptidyl tRNA is not. Given that the net effect of the peptidyl transferase reaction is the destruction of a high energy ester bond and the creation of a lower energy peptide bond, the forward direction of the peptide formation is strongly favored thermodynamically.

This description of the peptidyl transferase reaction raises as many questions as it answers. What keeps nascent peptides from inhibiting their own synthesis by filling up the PTC? How are discharged tRNAs removed from the P site? How does the peptidyl tRNA product of the peptidyl transferase reaction move from the A site, where it forms, to the $\mathrm{P}$ site, where it must reside if another amino acid is to be added to the nascent peptide chain? How is the next aminoacyl tRNA delivered to the A site?

Product clearance is thought to be the simplest of these issues. As nascent peptides form, they insert into a cavity called the peptide exit tunnel, which extends from the back of the PTC all the way through the body of the large ribosomal subunit [32]. It is not until the length of nascent peptides exceeds about 40 amino acids that their aminoterminal sequences emerge on the far side of the ribosome and start engaging with the apparatus that ensures protein folding and/or export. As far as we know, nascent polypeptides diffuse down the tunnel in response to the nudge they are given as each peptide bond forms, but there are hints that it may be more interesting [33]. Two recent crystal structures have provided insights into how ribosomes carrying completed proteins are recognized and the ester bond linking the protein to tRNAs is hydrolyzed, which is the ultimate step in product clearance [34,35].

The tRNA movements that reset the PTC after each round of peptide bond formation are still only partially understood [36]. Discussions of this process, which is called translocation, are best begun by reminding the reader that tRNAs are L-shaped RNAs that vary considerably in sequence but are nearly identical in shape. One arm of the $\mathrm{L}$, the acceptor stem, includes the $3^{\prime}$ terminal CCA sequence mentioned earlier. The other arm, the anticodon stem, terminates with a loop that includes an anticodon, which is the $3^{\prime}$-nucleotide sequence that pairs with mRNA codons during protein synthesis. (Aminoacyl tRNA synthetases ensure that the amino acids that get esterified to the acceptor stems of tRNAs are the ones encoded by mRNA triplets complementary to the anticodon sequences of those tRNAs.) Messenger RNAs bind to the small ribosomal subunit in the region where its head joins its body (Figure 1), and the place on the small subunit where tRNA anticodons interact with mRNA codons is called the decoding center. The A site and the $\mathrm{P}$ site of the decoding center are the locations where the anticodons of aminoacyl tRNAs and peptidyl tRNAs, respectively, are bound to the small ribosomal subunit just before peptide transfer occurs. Thus, translocation must reposition tRNAs on both ribosomal subunits. (tRNAs are L-shaped because there is a prominent ridge on the large subunit separating the PTC from the decoding center of the small subunit that only an L-shaped molecule can surmount.)

Translocation on the large subunit precedes translocation on the small subunit [37], and it seems to be a spontaneous, diffusive process. After peptide bond formation, the acceptor stems of both tRNAs in the PTC move towards the L1 arm of the large ribosomal subunit. The CCA sequence of the discharged tRNA moves from the P site of the PTC to the so-called E (exit) site of the large ribosomal subunit, which can bind only deacylated tRNAs [20], and the CCApeptide moiety of the peptidyl tRNA in the A site moves to the P site of the PTC. The A-to-P motion of peptidyl tRNAs is accompanied by a $180^{\circ}$ rotation of CCA sequences 
relative to the bodies of tRNAs. Large-subunit translocation correlates with a rotation of about $10^{\circ}$ of the small subunit relative to the large in the direction of the L1 arm, which is called ratcheting. The ratchet motion also seems to result from thermal diffusion, and it is unclear how tightly ratcheting is coupled to large-subunit translocation. Nevertheless, the data suggest that both must occur before small subunit translocation can take place.

Large-subunit translocation leaves the ribosome in a hybrid state, in the sense that the acceptor stem of the peptidyl tRNA is in a P site of the PTC while its anticodon end occupies the A site of the decoding center; the acceptor stem of the discharged tRNA is in the large-subunit $\mathrm{E}$ site while its anticodon is in the $\mathrm{P}$ site of the decoding center $[38,39]$ (Figure 4). Small-subunit translocation has two results. First, it advances the ribosome by three nucleotides along the mRNA to which it is bound in the $3^{\prime}$ direction, which places a new codon in the A site of the decoding center. Second, it resolves the hybrid state by making the anticodon end of peptidyl tRNA move from the A site to the $\mathrm{P}$ site of the decoding center, and the anticodon end of the deacylated tRNA move from the P site of the decoding center to the $\mathrm{E}$ site of the small subunit. The anticodon of the tRNA that moves from the A site to the $\mathrm{P}$ site of the decoding center remains associated with its codon in the mRNA so that the register in which the mRNA is being translated is maintained. It is unclear whether the anticodons of tRNAs in the E site actually interact with mRNA or not; there are biochemical data indicating they do, but the structural data are ambiguous.

Although small-subunit translocation can occur spontaneously, the spontaneous process is painfully slow. Like all the major steps in protein synthesis, it is catalyzed in the cell by a $\mathrm{G}$ protein. The $\mathrm{G}$ protein in this case, which is EF-G in bacteria and EF-2 in eukaryotes, binds to the ribosome with a GTP bound that is hydrolyzed in the process [40]. EM images of EF-G/ribosome complexes, which are all we have, show that this tadpole-shaped molecule binds to the ribosome with its head (which includes its GTPase site) bound to the large ribosomal subunit at the base of the L11 arm. That part of the EF-G binding site includes the sarcinricin loop (SRL) of 23S/28S rRNA (Figure 3) that, for reasons still unclear, is critically important for the activity of all of the G-protein factors that interact with the ribosome during protein synthesis. The distal end of the tail of the tadpole inserts into the A site of the decoding center of the small subunit. The binding of all proteins that interact with the EF-G binding site is promoted by the L10 complex and the rest of the L11 arm, but the details remain to be worked out.

In solution the GTPase activity of EF-G is very low, but it increases dramatically when the factor binds to the ribosome. Thus, shortly after EF-G.GTP binds to the ribosome, its GTP hydrolyzes. This causes EF-G to undergo a major conformational change that seems to push the anticodon stems of tRNAs across the decoding center, dragging the mRNA with them. Two additional events ensue: the ribosome unratchets; and EF-G.GDP is released into solution. Biochemical data suggest, and EM structures confirm, that the conformational changes that accompany EF-Gassisted translocation are more complicated than this account of translocation seems to require, but until the relevant atomic resolution structures become available, we are unlikely to understand them properly.

Once translocation is complete, the ribosome is ready to bind a new aminoacyl tRNA, and if there is a deacylated tRNA in the E site of the ribosome, aminoacyl tRNA binding is accompanied by release of that tRNA into solution. Biochemical data suggest that these two processes interact with each other, and structural data show that tRNA release correlates with conformational changes in the L1 arm of the large subunit.

Aminoacyl tRNAs are delivered to the ribosome by a second G protein, which is called EF-Tu in bacteria and EF- $1 \alpha$ in eukaryotes. The complex that EF-Tu forms with aminoacyl tRNA (and GTP), the so-called ternary complex, resembles EF-G in its overall shape, with the anticodon stem of the ternary complex corresponding to the tail of the EF-G tadpole and its EF-Tu/acceptor stem portion resembling the head. As far as we know, the ternary complex binds to the ribosome the same way that EF-G.GTP does. Its EF-Tu/ acceptor stem portion associates with the SRL region of the large subunit and its anticodon stem extends into the A site of the decoding center.

If the anticodon of the tRNA in a ternary complex base-pairs properly with the mRNA sequence in the A site of the decoding center, in other words if the codon and anticodon are cognate, a conformational change occurs that stimulates GTP cleavage and release of EF-Tu - GDP from the ribosome. The aminoacyl tRNA left behind is oriented so that its aminoacyl end is far from the A site of the PTC; the large reorientation required to place its acceptor stem in the PTC is called accommodation. Once accommodation has occurred, the system is ready for the formation of the next peptide bond, which ensues quickly thereafter.

\section{How the accuracy of decoding is ensured}

The binding of cognate aminoacyl tRNAs to the ribosome is the rate-limiting step in protein synthesis, and from the point of view of information transfer, it is the most important [41]. The cytoplasm of the average cell contains about 
60 different species of aminoacyl tRNAs. Given that diffusion is the process that brings ternary complexes to the ribosome, for every encounter with a cognate complex that results in accommodation there will be many encounters with non-cognate ternary complexes that must not result in accommodation if mRNAs are to be translated correctly. The reason is that once the wrong aminoacyl tRNA enters the A site of the PTC, the wrong amino acid will be inserted into the nascent protein: the PTC does not discriminate. The only way in which tRNA selection can lead to accurate translation is if the ribosome binds cognate complexes much more tightly than non-cognate complexes, and it does. The question is why.

In the end, ternary complex selection depends on basepairing between the sequence of the codon in the A site of the decoding center and tRNA anticodon sequences. However, it has been realized for decades that the difference in free energy between the formation of a perfect Watson-Crick helix three base-pairs long and the formation of a three-base-pair duplex in which one of the base juxtapositions is non-canonical is not large enough to explain the accuracy of translation.

The crystal structures and EM structures have both provided important insights into this aspect of protein synthesis [42]. We know from crystal structures of tRNAs bound to the ribosome in the accommodated state that when a cognate interaction occurs in the A site of the decoding center, the conformation of the small subunit changes so that two of its RNA bases form base-triples with the first two base-pairs of the codon-anticodon helix that form in that center. These interactions, which strongly stabilize the two base-pairs in question, cannot occur unless the bases juxtaposed in that helix form Watson-Crick pairs. For that reason, as had long been suspected, the difference in free energy between cognate pairing and non-cognate pairing is much bigger on the ribosome than it is in solution.

The orientation of the anticodon stem of a tRNA in the preaccommodation state is very different from its orientation after it has accommodated. What is going on in the decoding center at that point in the process? This question has been answered by EM images that show that before accommodation, the anticodon stems of tRNAs bend in such a way that their anticodon sequences will interact with the decoding site the same way they do after accommodation. Thus, the response of the A site of the decoding center to anticodons appears to be the same no matter whether the tRNA carrying them is in the pre- or postaccommodation conformation.

A vitally important consequence of the conformational change just alluded to is that, by some mechanism we do not understand, it activates the GTPase activity of EF-Tu. Thus, if a cognate interaction has occurred in the decoding center, the EF-Tu in a ternary complex will quickly hydrolyze its GTP and leave the ribosome so that the aminoacyl tRNA can accommodate. If the interaction between codon and anticodon is non-cognate, GTP hydrolysis is much slower, and the probability is correspondingly high that the non-cognate ternary complex will diffuse away from the ribosome intact, before GTP hydrolysis occurs.

Accommodation itself also contributes to the fidelity of protein synthesis. Suppose that against the odds, a noncognate ternary complex delivers its aminoacyl tRNA to the ribosome in the same way that a cognate complex does. Before that aminoacyl tRNA can engage in peptide-bond formation, it must accommodate and, as far as we know, the only interactions that keep accommodating aminoacyl tRNAs from diffusing away from the ribosome are their codonanticodon interactions. If that interaction is non-cognate, it will not be stabilized by interactions with the small subunit. It follows that non-cognate aminoacyl tRNAs are more likely to fall off the ribosome during accommodation than cognate aminoacyl tRNAs. Thus, base-pairing is used to discriminate cognate from non-cognate aminoacyl tRNAs twice every time an aminoacyl tRNA is delivered to the A site of the PTC. The ribosome proof-reads, as had long been suspected.

The understanding of decoding that has emerged from the combination of structural and biochemical investigations is a spectacular example of what everyone hoped would happen once the structure of the ribosome was understood at atomic resolution. However, as the above account shows, our understanding of even that aspect of the elongation cycle remains incomplete. Among the many other mysteries still unsolved are the mechanisms of action of LepA, which is a protein factor similar to EF-G that promotes reverse translocation, an unexpected phenomenon believed to contribute to fidelity [43]; and the tmRNA system that rescues ribosomes that have become stuck when translating a broken mRNA molecule [44]. The devotees of the ribosome will not run out of interesting problems to investigate for a while yet.

\section{Acknowledgements}

My understanding of protein synthesis has been shaped by conversations with my colleagues, especially Thomas Steitz and Rachel Green. Nevertheless, the responsibility for all the statements made above rests with me. This work was supported by a grant from NIH (GM-022778).

\section{References}

I. Moore PB: The ribosome returns. Nature 1988, 331:223-227.

2. Yonath A, Mussig J, Tesche B, Lorenz S, Erdmann VA, Wittmann HG: Crystallization of the large ribosomal subunits from Bacillus stearothermophilus. Biochem Internat 1980, I:428-435. 
3. Trakanov SD, Yusupov MM, Agalarov SC, Garber MB, Rayazantsev SN, Tishenko SV, Shirokov VA: Crystallization of 70S ribosomes and $30 S$ ribosomal subunits from Thermus thermophilus. FEBS Lett 1987, 220:319-322.

4. Hope H: Cryocrystallography of biological macromolecules: a generally applicable method. Acta Crystallogr B 1988, 44:22-26.

5. Hope H, Frolow F, von Bohlen K, Makowski I, Kratky C, Halfori Y, Danz H, Webster P, Bartles KS, Wittmann HG, Yonath A: Cryocrystallography of ribosomal particles. Acta Crystallogr B 1989, B45:190-199.

6. Frank J: Three-dimensional Electron Microscopy. New York: Oxford University Press; 2006.

7. Frank J, Zhu J, Penczek P, Li Y, Srivastava S, Verschoor A, Rademacher M, Grassucci R, Lata RK, Agrawal RK: A model for protein synthesis based on cryo-electron microscopy of the $E$. coli ribosome. Nature 1995, 376:44I-444.

8. Schlünzen F, Hansen HAS, Thygesen J, Bennett WS, Volkmann N, Levin I, Harms J, Bartles H, Zaytzev-Bashan A, Berkovitch-Yellin Z, Yonath A, Sagi I: A milestone in ribosomal crystallography: the construction of preliminary electron density maps at intermediate resolution. Biochem Cell Biol 1995, 73:739-749.

9. Ban N, Freeborn B, Nissen P, Penczek P, Grassucci RA, Sweet R, Frank J, Moore PB, Steitz TA: A 9 A resolution X-ray crystallographic map of the large ribosomal subunit. Cell 1998, 93:1 I05ilis.

10. Shevack A, Gewitz HS, Hennemann B, Yonath A, Wittmann HG: Characterization and crystallization of ribosomal particles from Haloarcula marismortui. FEBS Lett 1985, 184:68-7I.

II. Ban N, Nissen P, Hansen J, Capel M, Moore PB, Steitz TA: Placement of protein and RNA structures into a 5 A map of the 50 S ribosomal subunit. Nature 1999, 400:84I-847.

12. Clemons WM Jr, May JLC, Wimberly BT, McCutcheon JP, Capel MS, Ramakrishnan V: Structure of a bacterial $30 \mathrm{~S}$ ribosomal subunit at 5.5 A resolution. Nature 1999, 400:833-840.

13. Cate JH, Yusupov MM, Yusupova GZ, Earnest TN, Noller HF: Xray crystal structures of $70 \mathrm{~S}$ ribosome functional complexes. Science 1999, 285:2095-2104.

14. Ban N, Nissen P, Hansen J, Moore PB, Steitz TA: The complete atomic structure of the large ribosomal subunit at $2.4 \mathrm{~A}$ resolution. Science 2000, 289:905-920.

15. Schlünzen F, Tocilj A, Zarivach R, Harms J, Gluehmann M, Janell D, Bashan A, Bartles H, Agmon I, Franceschi F, Yonath A: Structure of functionally activated small ribosomal subunit at 3.3 A resolution. Cell 2000, 102:615-623.

16. Wimberly BT, Brodersen DE, Clemons WM, Morgan-Warren RJ, Carter AP, Vonrhein C, Hartsch T, Ramakrishnan V: Structure of the 30 S ribosomal subunit. Nature 2000, 407:327-339.

17. Harms J, Schluenzen F, Zarivach R, Bashan A, Gat S, Agmon I, Bartels $H$, Franceschi F, Yonath A: High resolution structure of the large ribosomal subunit from a mesophilic eubacterium. Cell 200I, 107:679-688.

18. Yusupov MM, Yusupova GZ, Baucom A, Lieberman K, Earnest TN, Cate JHD, Noller HF: Crystal structure of the ribosome at 5.5 A resolution. Science 200I, 292:883-896.

19. Korostelev A, Trakhanov S, Laurberg M, Noller HF: Crystal structure of a $70 \mathrm{~S}$ ribosome-tRNA complex reveals functional interactions and rearrangements. Cell 2006, I26:1065-1077.

20. Selmer M, Dunham CM, Murphy FVI, Weixibaumer A, Petry S, Kelley AC, Weir JR, Ramakrishnan V: Structure of the 70S ribosome complexed with mRNA and tRNA. Science 2006, 313:19351942.

21. Schuwirth BS, Borovinskaya MA, Hau CW, Zhang W, Vila-Sanjuro A, Holton JH, Doudna Cate JH: Structure of the bacterial ribosome at 3.5 A resolution. Science 2005, 310:827-834.

22. Ilag LL, Videler H, McKay AR, Sobott F, Fucini P, Nierhaus KH, Robinson CV: Heptameric (LI2)6/LIO rather than canonical pentameric complexes are found by tandem MS of intact thermopholic ribosomes Proc Natl Acad Sci USA 2005, 102:8192-8I97.

23. Karvan JM, Steitz TA: Structure of the base of the L7/I2 stalk of the Haloarcula marismortui large ribosomal subunit: analysis of LII movements. J Mol Biol 2007, 37I: 1047-I059.

24. Diaconu M, Kothe U, Schluenzen F, Fischer N, Harms JM, Tonevitskyu AG, Stark H, Rodnina MV, Wahl MC: Structural basis for the function of the ribosomal L7//2 stalk in factor binding and GTPase activation. Cell 2005, 121:991-1004.

25. Spahn CMT, Beckmann R, Eswar N, Penczek P, Sali A, Blobel G, Frank J: Structure of the $80 \mathrm{~S}$ ribosome from Saccharomyces cerevisiae - tRNA-ribosome and subunit-subunit interactions. Cell 200I, 107:373-386

26. Laurensen BS, Sorensen HP, Mortensen KK, Sperling-Petersen $\mathrm{HU}$ : Initiation of protein synthesis in bacteria. Microbiol Mol Biol Rev 2005, 69:101-123.

27. Simonetti A, Marzi S, Myasnikov AG, Fabbretti A, Yusupov M, Gualerzi CO, Klaholz BP: Structure of the 30S translation initiation complex. Nature 2008, 455:4I6-420.

28. Youngman EM, McDonald ME, Green R: Peptide release on the ribosome: mechanism and implications for translational control. Annu Rev Microbiol 2008, 62:353-373.

29. Hirokawa G, Demeshkina N, Iwakura N, Kaji H, Kaji A: The ribosome-recycling step: consensus or controversy? Trends Biochem Sci 2006, 31: | 43- I 49

30. Ribosome Structure and Function [http://www.mrc-Imb.cam.ac. uk/ribo/homepage/mov and overview.html]

31. Beringer M, Rodnina MV: The ribosomal peptidyl transferase. Mol Cell 2007, 26:3II-32I.

32. Voss NR, Gerstein M, Steitz TA, Moore PB: The geomtery of the ribosomal polypeptide exit tunnel. J Mol Biol 2006, 360:893-906.

33. Nakatogawa $\mathrm{H}$, Ito $\mathrm{K}$ : Intraribosomal regulation of expression and fate of proteins. ChemBioChem 2004, 5:48-5I.

34. Laurberg M, Asahara H, Korostelev A, Zhu J, Trakhanov S, Noller HF: Structural basis for translation termination on the $70 \mathrm{~S}$ ribosome. Nature 2008, 454:852-857.

35. Weixlbaumer A, Jin H, Neubauer C, Voorhees RM, Petry S, Kelley $A C$, Ramakrishnan V: Insights into translational termination from the structure of RF2 bound to the ribosome. Science 2008, 322:953-956.

36. Frank J, Gao H, Sengupta J, Gao N, Taylor DJ: The process of mRNA-tRNA translocation. Proc Natl Acad Sci USA 2007, 104: 1967I-19678.

37. Dorner S, Brunelle JL, Sharma D, Green R: The hybrid state of tRNA binding is an authentic translation elongation intermediate. Nat Struct Mol Biol 2006, 13:234-24 I.

38. Agirrezabala X, Lei JL, Brunelle JL, Ortiz-Meoz RF, Green R, Frank $\mathrm{J}$ : Visualization of the hydbrid state of tRNA binding promoted by spontaneous ratcheting of the ribosome. Mol Cell 2008, 32:190197.

39. Julian $\mathrm{P}$, Konevega AL, Scheres SHW, Lazaro M, Gil D, Wintermeyer W, Rodnina MV, Valle M: Structure of ratcheted ribosomes with tRNAs in hybrid states. Proc Natl Acad Sci USA 2008, 105:16922-16927.

40. Wilden B, Savelsbergh A, Rodnina MV, Wintermeyer W: Role and timing of GTP binding and hydrolysis during EF-G-dependent tRNA translocation. Proc Natl Acad Sci USA 2006, 103:1367013675 .

4I. Wintermeyer W, Peske F, Beringer M, Gromadski KB, Savelsbergh $A$, Rodnina M: Mechanisms of elongation on the ribosome: dynamics of a macromolecular machine. Biochem Soc Trans 2004, 32:733-737.

42. Ogle JM, Ramakrishnan V: Structural insights into translational fidelity. Annu Rev Biochem 2005, 74:129-177.

43. Evans RN, Blaha G, Bailey S, Steitz TA: The strucutre of LepA, the ribosomal back translocase. Proc Natl Acad Sci USA 2008, I05: 4673-4678.

44. Valle M, Gillet R, Kaur S, Henne A, Ramakrishnan V, Frank J: Visualizing tmRNA entry into a stalled ribosome. Science 2003, 300: 127-130.

45. Lake JA: Ribosome structure determined by electron microscopy of Escherichia coli small subunits, large subunits and monomeric ribosomes. J Mol Biol 1976, 105: I3 I-I59. 